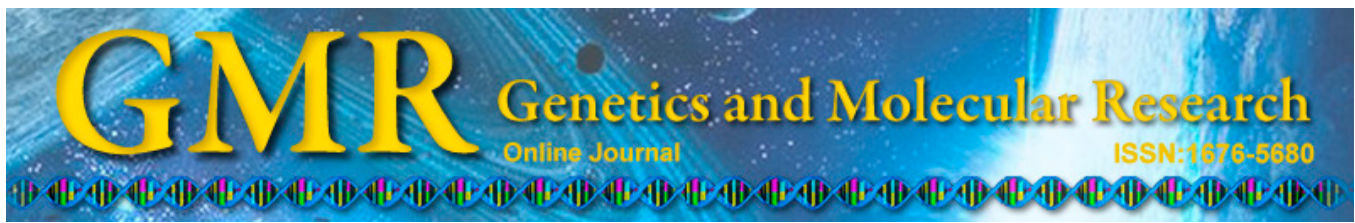

\title{
Characterization and expression of $D D X 6$ during gametogenesis in the Chinese mitten crab Eriocheir sinensis
}

\author{
Q. Li*, Y.L. Wang*, J. Xie, W.J. Sun, M. Zhu, L. He and Q. Wang \\ School of Life Science, East China Normal University, Shanghai, China \\ *These authors contributed equally to this study. \\ Corresponding authors: Q. Wang / L. He \\ E-mail: qwang@bio.ecnu.edu.cn / lhe@bio.ecnu.edu.cn
}

Genet. Mol. Res. 14 (2): 4420-4437 (2015)

Received June 23, 2014

Accepted April 15, 2015

Published April 30, 2015

DOI http://dx.doi.org/10.4238/2015.April.30.15

\begin{abstract}
DDX6 belongs to a family of DEAD-box RNA helicases, which are RNA splicing proteins that ensure the correct folding and structure of mature RNA. Gametogenesis requires the participation of many kinds of RNA. To explore its functions during Eriocheir sinensis gametogenesis, we cloned a full-length $D D X 6$ cDNA sequence from E. sinensis (Es-DDX6) which contains a 1536-nucleotide open reading frame encoding a 512-amino acid protein. Multiple sequence alignments showed that $E s-D D X 6$ has ten conservative DEAD-box family motifs. Tissue expression analysis of Es-DDX6 mRNA and protein levels showed that Es-DDX6 was highly expressed in both the ovary and testis. qRT-PCR analysis revealed the widespread expression of EsDDX6 mRNA during various stages of gonad development peaking in October. In addition, immunohistochemical studies showed that oocytes and the spermatogonium and primary spermatocytes of testes contained high levels of cytoplasmic Es-DDX6 and decreased expression levels in spermatids. Interestingly, there was no expression of $E s-D D X 6$ in these cells as they matured along the male reproductive system. Since oocytes and spermatocytes are active in meiosis and oocytes undergo
\end{abstract}


rapid growth in October, these results provide preliminary evidence that Es-DDX6 plays a role in E. sinensis gametogenesis and oocyte growth processes.

Key words: DDX6; Eriocheir sinensis; oocyte growth; gametogenesis

\section{INTRODUCTION}

Gametogenesis is a complicated process involving cellular transformations that result in the production of male and female haploid germ cells. Gametogenesis starts from the mitotic cell cycle followed by entry into meiosis and the completion of complex differentiation programs (Abou and Tulsiani, 2000; Hess and De Franca, 2009; Rangan et al., 2009; Hu and Peng, 2011). The spermatogonium or oogonium and sperm or ovum maturation processes require the concerted expression of a combination of functionally different and related genes as well as the participation of several types of RNA. Therefore, the stability of RNA is key to ensuring these processes function correctly. For example, mature oocytes and fertilized eggs are dependent on the posttranscriptional control of maternal mRNA until zygotic transcription is initiated (Ernoult et al., 2012).

RNA helicases are RNA splicing proteins involved in the folding and structural formation of mature RNAs. At the amino acid sequence level, RNA helicases are divided into the SFI and SFII superfamilies. Within the SFII superfamily, the Asp-Glu-Ala-Asp (DEAD) box protein family is currently the largest (Emery et al., 2004).

DEAD-box RNA helicases are found in almost all organisms and are involved in many mRNA metabolic process, including export, pre-mRNA splicing, translation initiation, decay, and ribosomal biogenesis. The DEAD-box family of proteins contain ten conserved structural motifs associated with ATP binding and hydrolysis, RNA binding, and helicase and RNA-unwinding activities. The conserved motifs are as follows: GaccPohlQ (Q motif), AxTGoGKT (motif I), PTRELA (motif Ia), TPGR (motif Ib), GG doublet, DEAD (motif II), SAT (motif III), IIFhxT+cx (motif IV), TRGIDIQAV (motif V), and HRIGRTGR (motif VI) (Cordin et al., 2006).

The DEAD-box family member DDX6 or RCK/p54 in mice has been shown to play significant roles in gametogenesis and early embryogenesis in eukaryotes (Abou and Tulsiani, 2000). In yeast, the DDX6 ortholog ste13 is essential for sexual reproduction (Maekawa et al., 1994), whereas the Caenorhabditis elegans ortholog CGH-1 is required for gametogenesis and provides protection from physiological germline apoptosis (Navarro et al., 2001). The Xenopus laevis, Spisula solidissima, and Drosophila melanogaster orthologs $\mathrm{Xp} 54, \mathrm{p} 47$, and Me13B, respectively, are integral components of stored (maternal) messenger ribonucleoprotein particles (mRNP) in oocytes (Ladomery et al., 1997; Minshall et al., 2001; Nakamura et al., 2001). Since Eriocheir sinensis has a close evolutionary relationship with $D$. melanogaster, we hypothesize that it plays a similar role in the regulation of gametogenesis. However, DDX6 research has mainly focused on some model organisms and its role in decapods remains elusive.

The aim of this study was to investigate if the expression of DDX6 in E. sinensis testis is similar to other species and determine the function E. sinensis DDX6 in spermatogenesis and oogenesis. To address this, we cloned a full-length E. sinensis DDX6 (Es-DDX6) and assessed its evolutionary status. To gain further insight into the biological importance of DDX6 
in gametogenesis, we investigated the mRNA and protein expression levels of Es-DDX6 in the male and female reproduction systems. Taken together, our results suggest that Es-DDX6 plays important roles in both female and male germ cells and provides the basis for artificially culturing crab.

\section{MATERIAL AND METHODS}

\section{Animals and sample collection}

Healthy and sexually mature adult Chinese mitten crabs of similar size were collected from the Jinshan Aquaculture Farm (Shanghai, China) from June 2012 to January 2013. Prior to the experiment, crabs were bred in filtered and aerated freshwater to acclimatize them to the new conditions. Prior to sacrifice, the crabs were placed in an ice bath for 3-5 min to lightly anesthetize them. Tissues (brain, hepatopancreas, heart, muscle, stomach, ovaries, testes, accessory gland, and seminal vesicle) were dissected and immediately frozen in liquid nitrogen and stored at $-80^{\circ} \mathrm{C}$.

\section{RNA extraction and rapid-amplification of cDNA ends (RACE) cloning}

Total RNA was extracted from the tissue samples using TRIzol reagent (RNA Extraction Kit, Invitrogen, USA) according to manufacturer instructions. The quality of the RNA was assessed by agarose gel electrophoresis and a NanoDrop 2000 Spectrophotometer (Thermo Fisher Scientific, USA). The $\mathrm{A}_{260} / \mathrm{A}_{280}$ ratio of the RNA ranged from 1.8 to 2.0.

Complimentary DNA (cDNA) was synthesized using an RNA reverse transcription kit (SMART ${ }^{\mathrm{TM}}$ cDNA kit, Clonetech, USA) and was used as the template for RACE. A partial $E s-D D X 6$ sequence was obtained from the expression sequence tag transcriptome data derived from the E. sinensis testis (He et al., 2012). First, we verified the sequence obtained from the transcriptome data. Polymerase chain reaction (PCR) primers were designed against the transcriptome fragment using the Primer Premier500 software (Table 1). The PCR program was set as follows: $94^{\circ} \mathrm{C}$ for $1 \mathrm{~min}, 35$ cycles of $94^{\circ} \mathrm{C}$ for $30 \mathrm{~s}, 57^{\circ} \mathrm{C}$ for $30 \mathrm{~s}, 72^{\circ} \mathrm{C}$ for $30 \mathrm{~s}$, and $72^{\circ} \mathrm{C}$ for $10 \mathrm{~min}$ for the final extension. To obtain the complete cDNA sequence of Es-DDX6, RACE technology was used to amplify the 5' and 3' cDNA ends according to the SMARTer ${ }^{\text {TM }}$ RACE cDNA Amplification Kit User Mannual (Clontech, Japan). The $3^{\prime}$ and 5' gene-specific RACE primers (Table 1) were designed based on confirmed sequences and chemically synthesized by Shanghai Invitrogen Biotech Co., Ltd. (Shanghai, China). Touchdown PCR was performed and the following program used: 5 cycles of $94^{\circ} \mathrm{C}$ for $30 \mathrm{~s}, 72^{\circ} \mathrm{C}$ for $3 \mathrm{~min}$; 5 cycles of $94^{\circ} \mathrm{C}$ for $30 \mathrm{~s}, 68^{\circ} \mathrm{C}$ for $30 \mathrm{~s}, 72^{\circ} \mathrm{C}$ for $3 \mathrm{~min}, 28$ cycles of $94^{\circ} \mathrm{C}$ for 30 $\mathrm{s}, 65^{\circ} \mathrm{C}$ for $30 \mathrm{~s}, 72^{\circ} \mathrm{C}$ for $3 \mathrm{~min}$, and $72^{\circ} \mathrm{C}$ for $7 \mathrm{~min}$ for the final extension step. The PCR products were separated by agarose gel electrophoresis and DNA gel green was added to visualize the bands. The PCR products of the expected size were extracted and purified with TaKaRa MiniBEST DNA Fragment Purification Kit version 4.0 (TaKaRa, Japan). Purified PCR products were cloned into the PMD19T vector (TaKaRa) and transformed into competent Escherichia coli TOP-10 cells. Recombinants were identified through blue/white color selection and the corresponding colonies picked and used directly to confirm the presence of the insert by PCR. Positive clones were sequenced using SP6 and T7 primers (Table 1) by Majorbio (Shanghai, China). 


\begin{tabular}{ll} 
Table 1. Primer sequences used in the study. & \\
\hline Primer name & Sequence \\
\hline Degenerate primers for target Es-DDX6 & \\
Forward primer & 5'-CCCTCAGCGGACGAGACATT-3' \\
Reverse primer & 5'-GTTCCCTAACCAGCCCCTAAAT-3' \\
qRT-PCR primers & \\
$D D X 65$ 'primer & 5'-TTTCCCAAAATGGCTGAGACC-3' \\
$D D X 6$ 3'primer & 5'-AGGGCAAAGCGGTCATCGTA-3' \\
$\beta$-actin and GAPDH for qRT-PCR & \\
$\beta$-actin-F & 5'-CTCCTGCTTGCTGATCCACATC-3' \\
$\beta$-actin-R & 5'-GCATCCACGAGACCACTTACA-3' \\
GAPDH-F & 5'-TGGTGGAGCCAAGAAGGTG-3' \\
GAPDH-R & 5'-ACGGGAGCCAGGCAGTT-3' \\
Sequencing & 5'-TAATACGACTCACTATAGG-3' \\
T7 & 5'-ATTTAGGTGACACTATAGAA-3' \\
SP6 & \\
\hline
\end{tabular}

\section{Sequence alignments and phylogenetic analysis}

The BLAST program at the National Center for Biotechnology Information (NCBI; http://www.ncbi.nlm.nih.gov/blast) was used to analyze sequence similarities. The open reading frame (ORF) of the DDX6 cDNA was determined using ORF Finder (www.ncbi.nlm.nih. gov/gorf/) and the corresponding amino acid sequence was ascertained. The isoelectric point, theoretical mass, active sites, and functional domains of the protein were analyzed at http:// web.expasy.org/compute_pi and http://prosite.expasy.org/. DDX6, DDX4, DDX25, DDX52, and DDX20 amino acid sequences from various species of vertebrates and invertebrates were obtained from the NCBI GenBank database (Table 2) and then aligned with the full-length EsDDX6 sequence using the ClustalX Multiple Alignment program (Assumpção et al., 2008). SMART (Simple Modular Architecture Research Tool; http://smart.embl-heidelber.de/) and SignalP4.1 (http://www.cbs.dtu.dk/services/SignalP) were used to predict homologous conserved domains and signal peptides, respectively. The phylogenetic tree was constructed according to the neighbor-joining method of the MEGA version 4.0 software (Kumar et al., 2004) using bootstrap resampling (500 pseudo-replicates).

\section{Real-time quantitative (qRT) PCR of DDX6 gene expression}

First strand cDNA was synthesized using the PrimeScript ${ }^{\mathrm{TM}}$ Real-time PCR Kit (TaKaRa, Japan) and was used as the template for the qRT-PCR analysis using the CFX96TM Real-Time System (Bio-Rad). Each reaction contained 12.5 $\mu \mathrm{L}$ 2X SYBR premix Ex Taq (TaKaRa, Japan), $1 \mu \mathrm{L}$ cDNA template $(100 \mathrm{ng} / \mu \mathrm{L}), 9.5 \mu \mathrm{L}$ PCR-grade water, and $1 \mu \mathrm{L} 10$ $\mu \mathrm{M}$ primer pair solution. The qRT-PCR primers (DDX6 5' primer and DDX6 3 ' primer; Table 1) were designed based on the cloned $E s-D D X 6$ cDNA to produce a 107-bp amplicon. All qRT-PCR reactions were run in triplicate using independently extracted RNA samples and both $\beta$-actin and GAPDH served as constitutive controls. The following qRT-PCR program was used: $95^{\circ} \mathrm{C}$ for $30 \mathrm{~s}, 40$ cycles of $95^{\circ} \mathrm{C}$, and a $0.5^{\circ} \mathrm{C} / 5 \mathrm{~s}$ incremental increase from $60^{\circ} \mathrm{C}$ to $95^{\circ} \mathrm{C}$ that lasted for $30 \mathrm{~s}$ per cycle. Data from the three replicate samples were analyzed using CFX Manager TM software (version 1.0). The change in cycle threshold ( $\Delta \mathrm{Ct}$; difference in the $\mathrm{Ct}$ values between target and control genes) for each sample was subtracted from that of the calibrator. Every $\Delta \mathrm{Ct}$ minus the lowest $\Delta \mathrm{Ct}$ (the $\Delta \Delta \mathrm{Ct}$ value) and $D D X 6$ expression level 
Table 2. Amino acid sequences used for constructing phylogenic tree and multiple sequence alignments.

\begin{tabular}{|c|c|c|}
\hline Species & GenBank accession No. & Protein \\
\hline Danio rerio & E7FD91 & DDX6 \\
\hline Xenopus laevis & P54824 & DDX6 \\
\hline Homo sapiens & P26196 & DDX6 \\
\hline Mus musculus & P54823 & DDX6 \\
\hline Drosophila melanogaster & NP_523533.2 & DDX6 \\
\hline Oreochromis niloticus & XP_003447300.1 & DDX6 \\
\hline Ciona intestinalis & XP_002123307.1 & DDX6 \\
\hline Acromyrmex echinatior & EGI 60783.1 & DDX6 \\
\hline Camponotus floridanus & EFN61728.1 & DDX6 \\
\hline Acyrthosiphon pisum & XP_001951330.2 & DDX6 \\
\hline Takifugu rubripes & XP_003968632.1 & DDX6 \\
\hline Myotis brandtii & XP_005856840.1 & DDX6 \\
\hline Dugesia japonica & BAF 57607.1 & DDX6 \\
\hline Tribolium castaneum & XP_974536.1 & DDX6 \\
\hline Nasonia vitripennis & XP_001602897.1 & DDX6 \\
\hline Bombyx mori & XP_004925561.1 & DDX6 \\
\hline Echinops telfairi & XP_004712835.1 & DDX6 \\
\hline Ovis aries & XP_004016131.1 & DDX6 \\
\hline Ornithorhynchus anatinus & XP_001516184.2 & DDX6 \\
\hline Tupaia chinensis & XP_006152352.1 & DDX6 \\
\hline Homo sapiens & Q9N̄QI0 & DDX4 \\
\hline Mus musculus & Q61496 & DDX4 \\
\hline Danio rerio & A1L1Z2 & DDX4 \\
\hline Crepidula fornicate & D9I032 & DDX4 \\
\hline Branchiostoma belcheri & D3YII5 & DDX4 \\
\hline Drosophila melanogaster & P09052 & DDX4 \\
\hline Tribolium castaneum & D6WSI7 & DDX4 \\
\hline Eriocheir sinensis & ADM64419.1 & DDX4 \\
\hline Homo sapiens & Q9UHI6 & DDX20 \\
\hline Danio rerio & P0C218 & DDX20 \\
\hline Mus musculus & Q9JJY4 & DDX20 \\
\hline Rattus norvegicus & F1MAM8 & DDX20 \\
\hline Xenopus tropicalis & F6ZDW4 & DDX20 \\
\hline Rattus norvegicus & Q9QY16 & DDX25 \\
\hline Mus musculus & Q9QY15 & DDX25 \\
\hline Homo sapiens & Q9UHL0 & DDX25 \\
\hline Xenopus tropicalis & F6TZS6 & DDX25 \\
\hline Homo sapiens & Q9Y2R4 & DDX52 \\
\hline Mus musculus & Q8K301 & DDX52 \\
\hline Rattus norvegicus & Q99PT0 & DDX52 \\
\hline Danio rerio & Q32PQ3 & DDX52 \\
\hline Oryzias latipes & H2LKQ7 & DDX52 \\
\hline
\end{tabular}

was estimated by $2^{-\Delta \Delta C t}$. Expression level data were subsequently analyzed by Microsoft Excel and the SPSS software (version 20.0) (Zhao et al., 2007) and reported as means \pm standard deviation. Statistical significance was evaluated using analysis of variance (ANOVA) and $\mathrm{P} \leq$ 0.05 was considered to be significant.

\section{Histological analysis of the $E$. sinensis reproductive system}

Testis, ovary, and seminal vesicle samples were fixed at room temperature in $4 \%$ (v/v) paraformaldehyde for $24 \mathrm{~h}$ before being dehydrated and embedded in paraffin (Du et al., 1988a). Slides were immersed in dimethylbenzene for 5-10 min, followed by absolute ethyl alcohol for $5 \mathrm{~min}, 90 \%$ ethanol for $2 \mathrm{~min}, 70 \%$ ethanol for $2 \mathrm{~min}$, and finally distilled water for $2 \mathrm{~min}$. Hematoxylin stain solution was applied for 5-10 min, rinsed in tap water for $10 \mathrm{~min}$ to remove excess dye, and treated with $95 \%$ ethanol for $5 \mathrm{~s}$. Eosin stain was added for 2 min, 
dehydrated in $95 \%$ ethanol for $5 \mathrm{~min}$, and then placed in dimethylbenzene for 5 min to make the sections transparent. Finally, the paraffin sections were sealed using Permount ${ }^{\mathrm{TM}}$ Mounting Medium.

\section{Western blotting}

The testis and ovary (October) tissues were taken out from $-80^{\circ} \mathrm{C}$ refrigerator and were ground with the liquid nitrogen. The ground sample was homogenized on ice for $30 \mathrm{~min}$ in Cell Lysis Solution (Beyotime) in the presence of a pre-cooled Protease Inhibitor Cocktail (TaKaRa, Japan) to prevent protein degradation. Homogenates were centrifuged at 12,000 $\mathrm{g}$ and supernatants were collected and stored at $-80^{\circ} \mathrm{C}$. Protein concentrations were determined using a BCA Protein Assay kit (Beyotime). Ten micrograms of protein were separated by electrophoresis on a $0.1 \%$ sodium dodecyl polyacrylamide gel electrophoresis (SDS-PAGE; $10 \%$ polyacrylamide) and transferred onto a polyvinylidene difluoride (PVDF) membrane. Membranes were blocked for $1 \mathrm{~h}$ in blocking solution [5\% (w/v) skimmed milk in TBS-T; 10X TBS: $12.1 \mathrm{~g}$ Tris, $17.5 \mathrm{~g}$ $\mathrm{NaCl}$, and $1 \mathrm{~mL}$ Tween-20 made up to $1 \mathrm{~L}$ with distilled water, $\mathrm{pH} 7.4]$ then immunoblotted overnight with anti-human DDX6 (1:500; ab103308), anti- $\beta$-actin (1:1000; M1210-5), and anti-GAPDH $(1: 2000 ; 10494-1-A P)$ antibodies. The anti-DDX6 antibody corresponds to internal amino acids 354-384 of human DDX6 (81\% homology with Es-DDX6). Blots were rinsed three times with TBS-T for $45 \mathrm{~min}$ and then incubated with the secondary antibodies (diluted 1:1000 in TBS) for $1 \mathrm{~h}$ at $37^{\circ} \mathrm{C}$. Blots were washed three times with TBS-T and antibody-antigen complexes detected using an enhanced chemiluminescence detection kit (CWBIO).

\section{Immunohistochemistry of DDX6 expression in the testis and ovary of $E$. sinensis}

Testis, ovary, seminal vesicle, and vas deferens samples (August) were fixed at room temperature in $4 \%(\mathrm{v} / \mathrm{v})$ paraformaldehyde for $24 \mathrm{~h}$ before dehydrating and embedding in paraffin. Paraffin slides were immersed (for $5 \mathrm{~min}$ at each step) twice in dimethylbenzene, twice in absolute ethyl alcohol, once in $95 \%$ ethanol, once in $85 \%$ ethanol, once in $75 \%$ ethanol, and once in deionized water. Antigen retrieval was achieved by immersing the slides in $10 \mathrm{mM}$ citrate buffer and boiling in a microwave oven for $10 \mathrm{~min}$. Slides were rinsed three times with phosphate-buffered saline (PBS) then prepared for immunohistochemistry analysis according to manufacturer instructions of the SP-9001/9002 Histostain-Plus Kits (SUNBIO). The slides were first incubated in $3 \% \mathrm{H}_{2} \mathrm{O}_{2}$ deionized water for 5-10 min to eliminate endogenous peroxidase activity and then rinsed three times with PBS. Slides were blocked with normal goat serum working fluid for 10-15 min at room temperature then incubated with polyclonal rabbit anti-DDX6 antibodies (diluted 1:100) overnight at $4^{\circ} \mathrm{C}$. Slides were then washed three times with PBS and then labeled with biotinylated goat anti-rabbit IgGs for $10-15$ min at $37^{\circ} \mathrm{C}$. Slides were rinsed three times with PBS and then treated with horseradish enzyme marker chain mold avidin working fluid (S-A/HRP) for 10-15 min at $37^{\circ} \mathrm{C}$. Slides were rinsed with PBS and immunoreactive proteins were visualized after the addition of 3,3'- $N$-diaminobenzidine tetrahydrochloride (CWBIO) for $1 \mathrm{~min}$. Slides were counterstained with hematoxylin for $3 \mathrm{~min}$, rinsed with water, dehydrated in a graded series of alcohol, transferred to xylene, and sealed with Permount ${ }^{\mathrm{TM}}$ Mounting Medium. For the negative control, normal IgGs were used in placed of the primary antibodies. Imaging was performed using a light microscope (Leica). 


\section{RESULTS}

\section{$E s-D D X 6$ cDNA sequence analysis and putative protein in $E$. sinensis}

The fragment sequence obtained from the transcriptome analysis of $E$. sinensis testis contains both ends of the Es-DDX6 gene (Figure 1); 5'RACE: 1000 bp, 3'RACE: 500 bp. The Es-DDX6 cDNA sequence is $1799 \mathrm{bp}$ with an ORF of 1536 bp that encodes a putative 512-amino acid protein. In addition to a 41-bp 5'-untranslated region (UTR), the fragment contains a 222-bp 3'-UTR with a 27 bp polyadenylation tail. The polyadenylation site (AATTTA) occurs 31 bp upstream from the 3'-terminal poly(A) (Figure 2). The complete Es-DDX6 sequence has been deposited in GenBank (GenBank ID: JX104651). The predicted theoretical mass and isoelectric point of the encoded protein is $58.1 \mathrm{kDa}$ and 6.26 , respectively. There is no signal peptide in Es-DDX6 and it is referred to as Es-DDX6. Secondary structure analysis indicates that Es-DDX6 contains the ten conservative motifs considered to be characteristic features of DEAD-box family members, including GWNMTTIFPQ, AKNGAGKT, PTRELA, GG, TPGR, DEAD, SAT, IIFCNSTQ, TRGIDIQAV, and HRIGRSGR (Figure 2), suggesting it may have ATPase, RNA-unwinding, RNA-binding, and helicase activities.

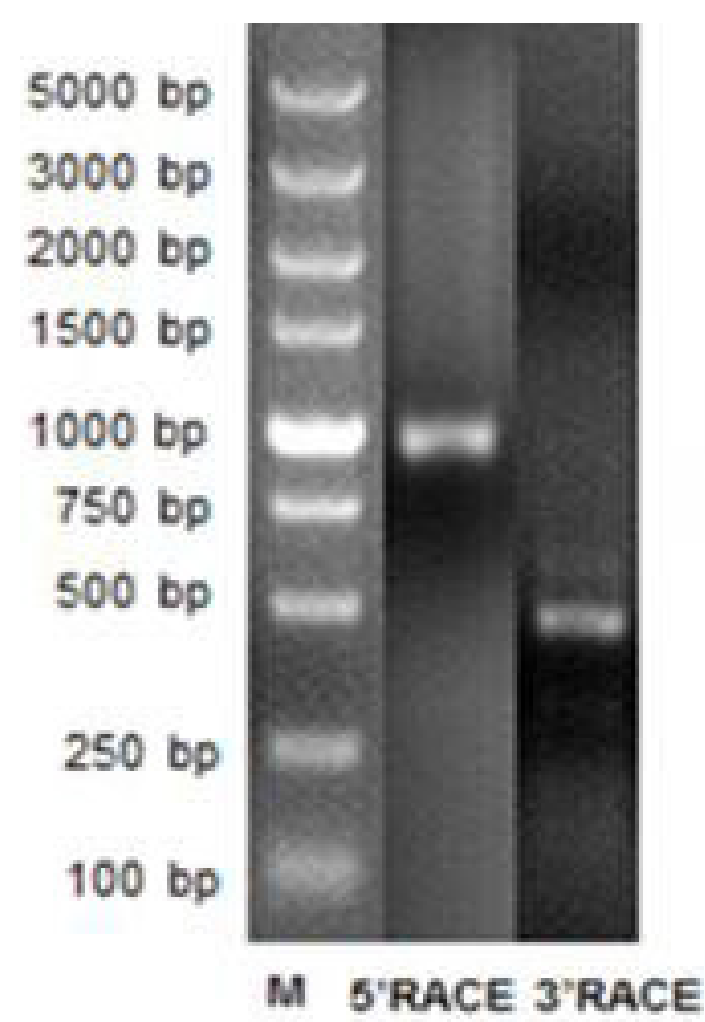

Figure 1. Target DDX6 fragments obtained by RACE PCR. Lane $M=$ DL5000 marker; 5'RACE: $\sim 1000$ bp; 3'RACE: 500 bp. 


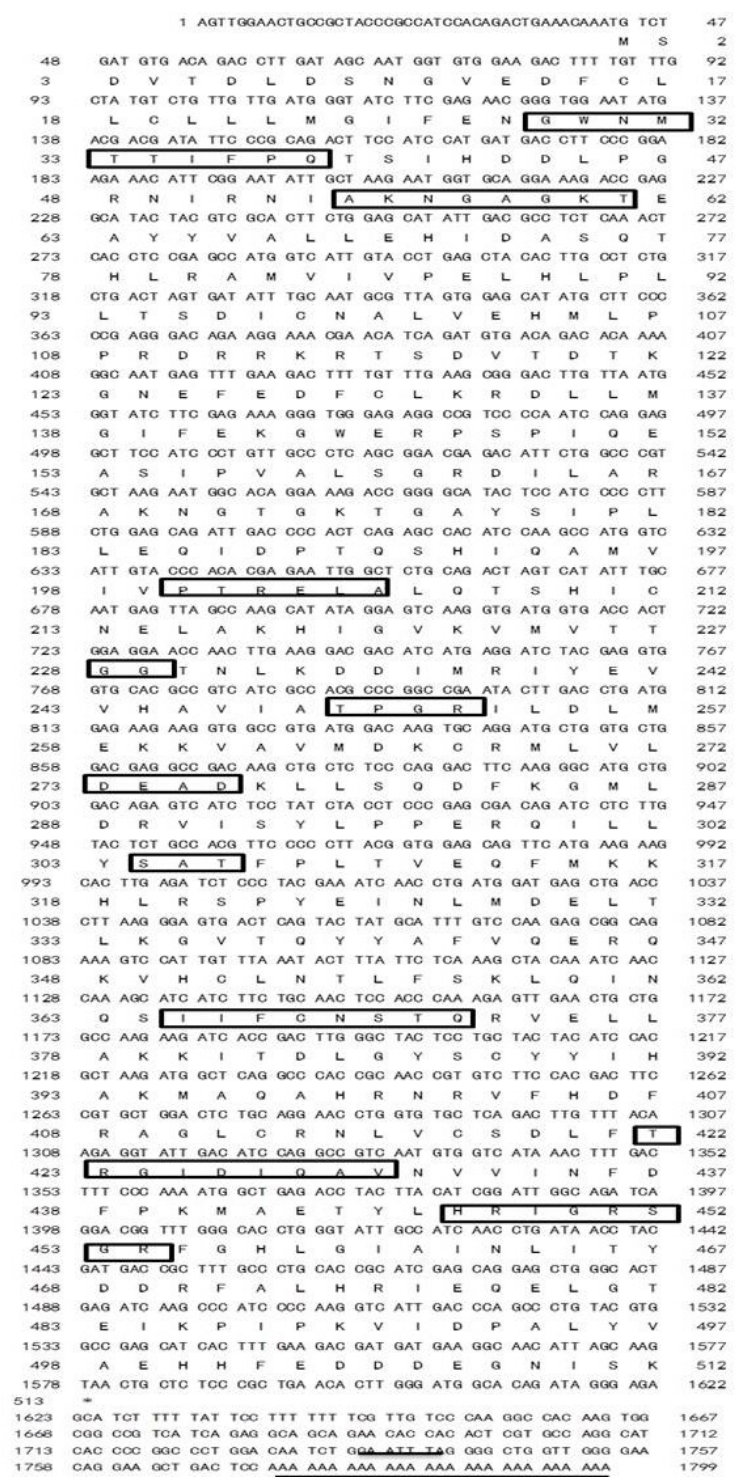

Figure 2. Nucleotide and predicted amino acid sequences of Es-DDX6. Residues 1-41 and 1581-1799 correspond to the 5'and 3'-UTRs, respectively. The conserved domains of DEAD-box family members are marked with black boxes. Asterisk indicates the stop codon. The polyadenylation signal is underlined. A polyadenylation site (AATTTA) is bold underlined.

\section{Multiple sequence alignment and phylogenetic analysis}

Sequence alignment of Es-DDX6 with DEAD-box RNA helicase family members confirms it is a member of a subfamily that includes human and mouse rck/p54, Drosophila ME31B, and X. laevis Xp54 (Figure 3A). While Es-DDX6 is evolutionarily related to both the Drosophila Me13B (81\%) and Homo sapiens (78\%) orthologs, it is most closely related to the Tribolium castaneum ortholog (89\%), suggesting conservation between DDX6 orthologs. 
All DEAD-box proteins share certain conserved motifs, alignment of the AxTGoGKT and HRIGRTGR motifs showed that DDX4, DDX25 and DDX52 have the same HRIGRTGR motif (Figure 3B), however, the HRIGRTGR motif of the DDX20 is HRIGRAGR, and the DDX6 is HRIGRSGR, which the sixth amino acid various between different subfamilies. In addition, the AxTGoGKT motif varied amongst the different DEAD-box family members, such as DDX4 is AQTGSGKT, DDX20 is AKSGTGKG, DDX25 is SQSGTGKT, DDX52 is APTGSGKT, and DDX6 is AKNGTGKT/S. The divergence within the motif is thought to confer functional specificity to many members of DEAD-box family, for example in the targeting of specific RNA sequences to RNA-binding domains. Therefore, it is very interesting to note the variety of the amino acids within the motif of different DEAD-box family members.

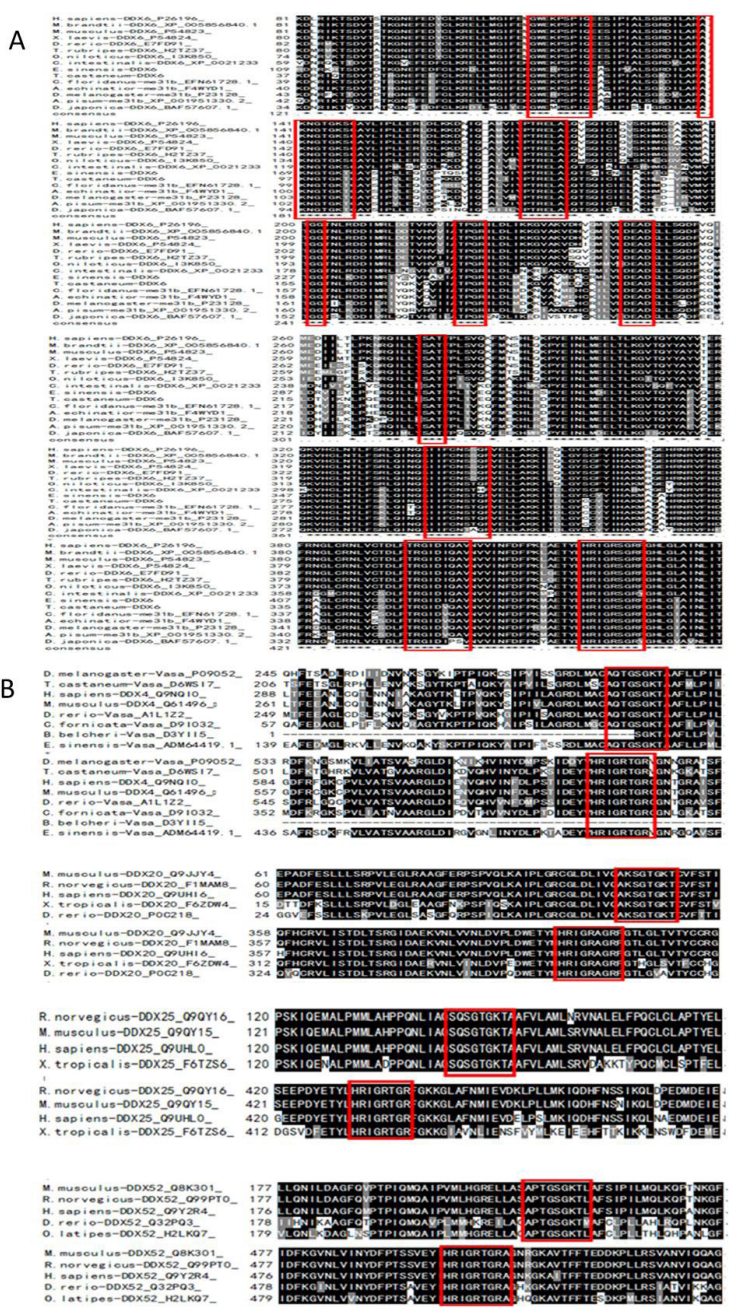

Figure 3. Multiple sequence alignment of Es-DDX6 with homologous amino acid sequences. (A) Sequence alignment of Es-DDX6 with DDX6 from other species. (B) Sequence alignment of DDX6, DDX4, DDX20, DDX25, and DDX52 from different species. Identical or similar residues are highlighted. Asterisk indicates identical, dot indicates very similar, colon indicates similar. Red boxes indicate conserved domains. 
Considering the variability of the AxTGoGKT and HRIGRSGR motifs, a phylogenetic tree was constructed to analyze the genetic relationship between $E s-D D X 6, D D X 6$ from other species, and other DEAD-box family members (Figure 4). In this tree, Es-DDX6 appears to cluster more closely with insect DDX6 homologs than with vertebrates. Many mammalian DDX6s grouped together, while the X. laevis homolog was independent. Danio rerio and Takifugu rubripes, two fish species, grouped within a cluster, further confirming the accuracy of Es-DDX6. Furthermore, $D D X 6$ and $D D X 25$ grouped together most closely, followed by $D D X 20$. As well, $D D X 52$ and $D D X 4$ grouped together, but were divided into the third subclusters, providing evidence that $D D X 6$ and $D D X 25$, and $D D X 52$ and $D D X 4$ may have functionally similar roles in physiological regulation.
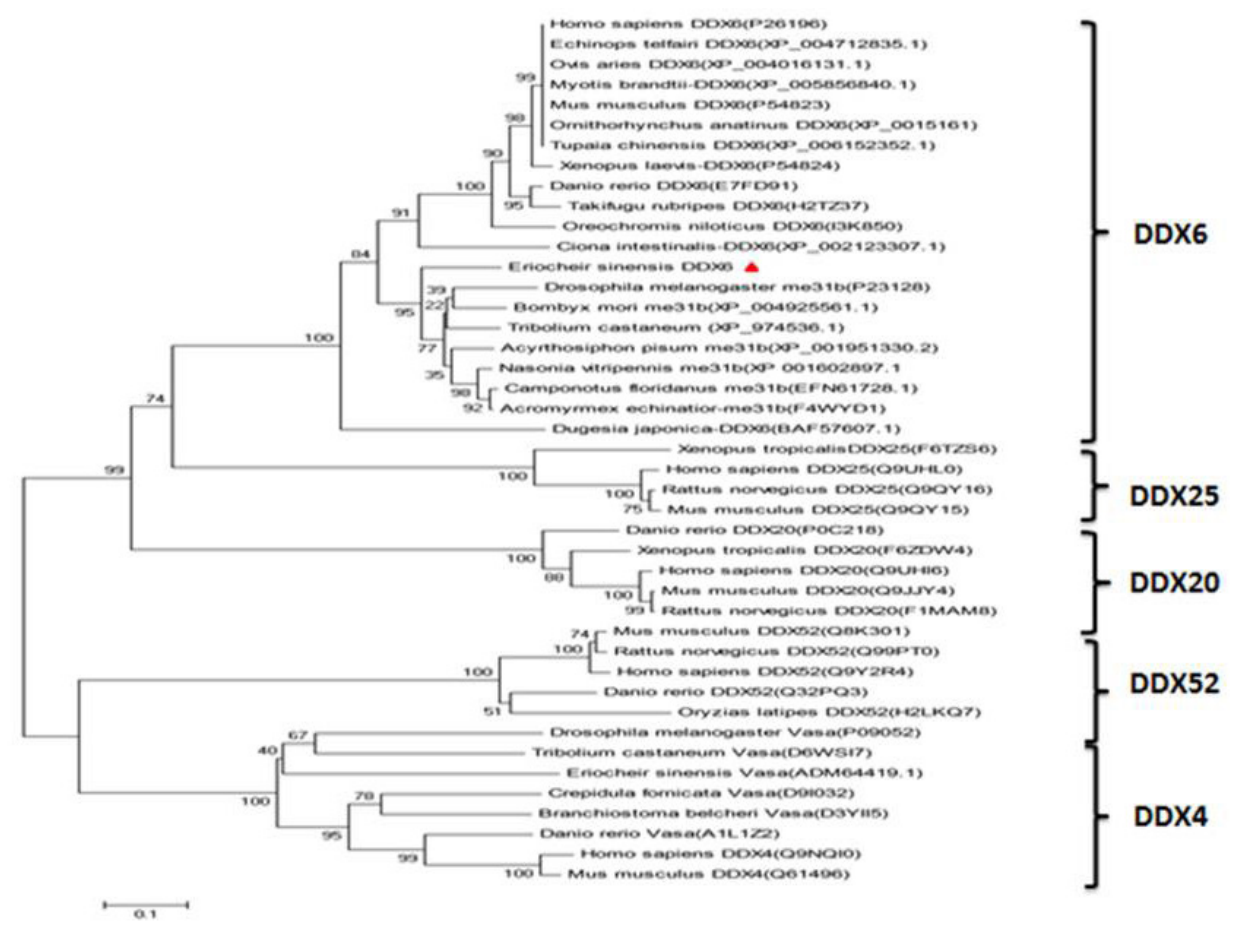

Figure 4. Phylogenetic tree based on amino acid sequences. Relationships between $E s-D D X 6$ and $D D X 6$ from other species, and between $D D X 6$ and other DEAD-box members, such as $D D X 25, D D X 52, D D X 20$, and $D D X 4$. The branch for $E s-D D X 6$ is marked with a red triangle. Scale bar represents a $10 \%$ divergence between sequences.

\section{Temporal and spatial expression analysis of DDX6 in E. sinensis}

Relative mRNA expression levels of Es-DDX6 in eight tissues (testis, heart, stomach, muscle, hemocytes, thoraces, intestine, and ovary) as well as different stages of gonad development from healthy crabs were measured by qRT-PCR using the $\beta$-actin and GAPDH genes as internal controls (Figures 5, 6, and 7). Es-DDX6 mRNA was expressed mainly in the gonad tissues (testes and ovary) with expression significantly greater in the testes. The other tissues were marked by extremely low levels of expression. These results provide preliminary evidence that Es-DDX6 may participate in the development of the ovary and testis. 


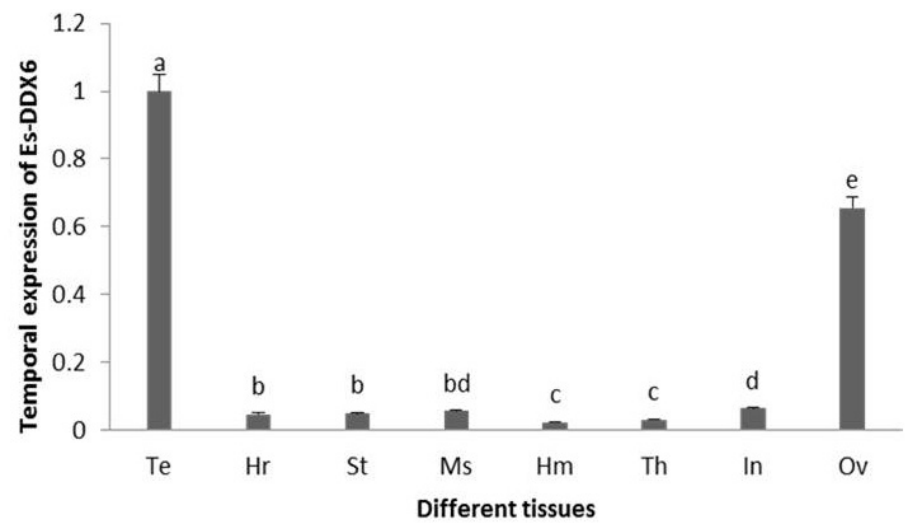

Figure 5. Relative expression levels of Es-DDX6 mRNA in different tissues. Te: testis; Hr: heart; St: stomach; Ms: muscle; Hm: hemocytes; Th: thoraces; In: intestine; Ov: ovary. Significant differences $(\mathrm{P} \leq 0.05)$ of different tissues were analyzed by one-way analysis of variance and are indicated by lowercase letters.

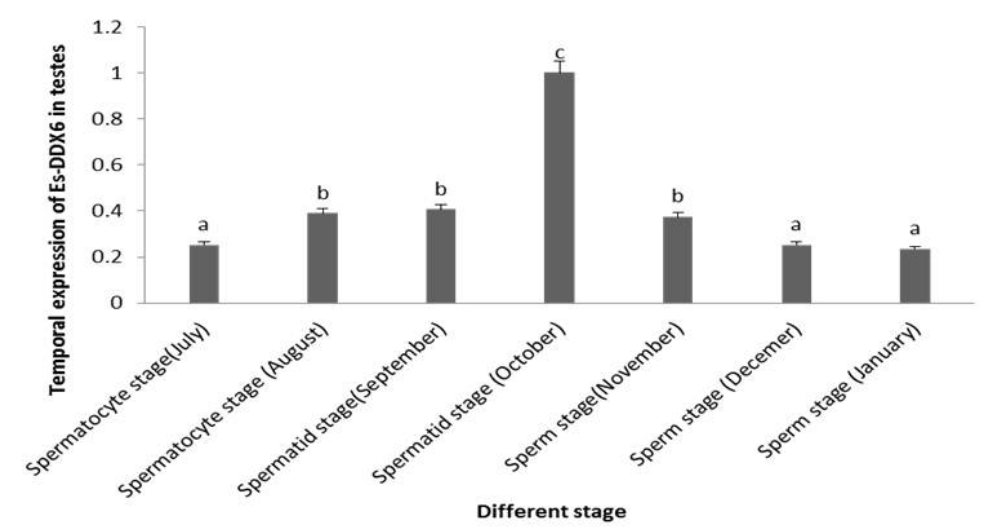

Figure 6. Relative expression levels of Es-DDX6 mRNA at different stages of testis development. Statistically significant differences are indicated with lower case letters $(\mathrm{P} \leq 0.05)$.

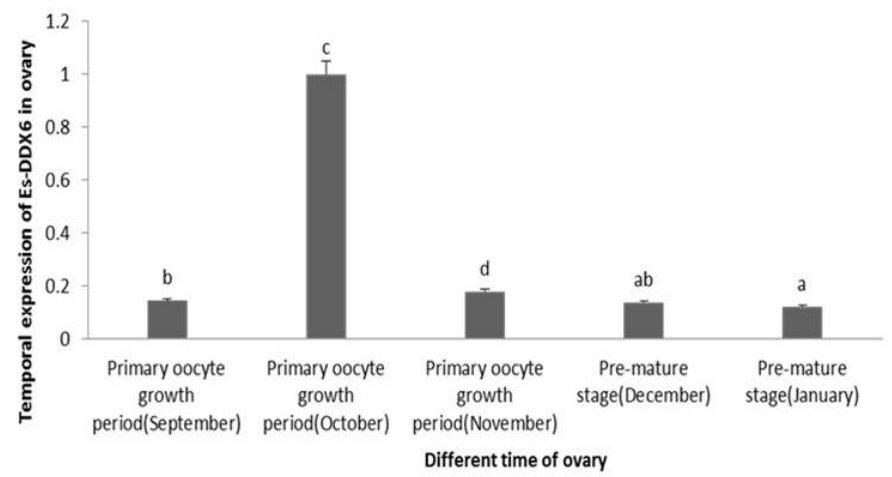

Figure 7. Relative expression levels of Es-DDX6 mRNA at different stages of ovary development. Statistically significant differences are indicated with lower case letters $(\mathrm{P} \leq 0.05)$. 
In order to explore the specific functions of Es-DDX6 in E. sinensis, we performed the following experiments. According to Du et al. (1988a), E. sinensis spermatogenesis occurs in the seminiferous tubules and is divided into five stages: Stage I, the spermatogonium stage (May to June); Stage II, the spermatocyte stage (Stage II-1: July; Stage II-2: August) ; Stage III, the spermatid stage (Stage III-1: September; Stage III-2: October); Stage IV, the sperm stage (Stage IV-1: November; Stage IV-2: December; Stage IV-3: January); and Stage V, the rest stage (April to May). Oogenesis is also divided into five stages: Stage I, the oogonium stage (April to June); Stage II, the primary oocyte niche (June to July) (Stage II-1: June; Stage II-2: July); Stage III, the primary oocyte growth period (August to November) (Stage III-1: August to September; Stage III-2: October to November); Stage IV, the pre-mature stage (December to March); Stage V, the mature stage (March to April). The mRNA expression level of $E s-D D X 6$ varied in a stage-dependent manner with a slight increase during the spermatocyte stage (July to August), followed by a significant increase during September to October. Expression levels notably decrease from October to November to levels similar to those found during the August to September period. During December and January, expression levels are quite low and similar to those observed in July. However, the expression of Es-DDX6 in ovaries during the primary oocyte growth period is significantly higher than in the pre-mature stage, with peak expressions seen in October (Figures 6 and 7). These data suggest that EsDDX6 may be involved in the oocyte growth process, however, why the expression levels of Es-DDX6 differ in the various stages of gonad development and why it peaks in October have yet to be elucidated.

\section{Histological analysis of the reproductive system and DDX6 protein expression in E. sinensis}

The E. sinensis male reproductive system consists of a pair of testes made up of raw seminiferous tubules, vas deferens, seminal vesicle, the accessory gland, and the ejaculatory duct. The male reproductive system of $E$. sinensis develops differently from other crustaceans and cells gradually mature from the end of testis to the vas deferens. The E. sinensis female gonad includes the ovary, spermatheca, and a very short fallopian tube. As the ovary matures, it separates into many oocyte development zones (Xue et al., 1987; Du et al., 1988b). The more detailed structures of the reproductive tissues were investigated with the aid of hematoxylineosin stained paraffin slides of the testis, ovary, and seminal vesicle samples. Examination of cross-sections of the seminiferous tubule in the testes showed that one side of this structure is the germinative area, while the other side is the epithelium. In the germinative area, there was germ cell proliferation and the cells are propelled gradually to the lumen (Figure 8A and B). However, spermatogenesis occurs in batches, and while some of the spermatogonia develop into sperm cells at different stages, the other spermatogonia remain in the resting state (the resting spermatogonia) to prepare for subsequent spermatogenesis. Therefore, in the same seminiferous tubule cross-section, most of the four different developmental stages were observed in the germinative area (Figure $8 \mathrm{C}$ ). Nevertheless, a few of the seminiferous tubules had only one germinative area (Figure 8D). Due to the spermatogenic wave, the germ cells in the seminiferous tubule are often in different developmental stages (Du et al., 1988a). The seminal vesicle is the storage site of spermatophores (Figure 8E and F). In October, the ovary consisted of many oocytes, with large nuclei and scattered nucleoli. In contrast, the nuclei of the follicular cells surrounding the oocyte were small and deeply dyed (Figure 8G and H). 

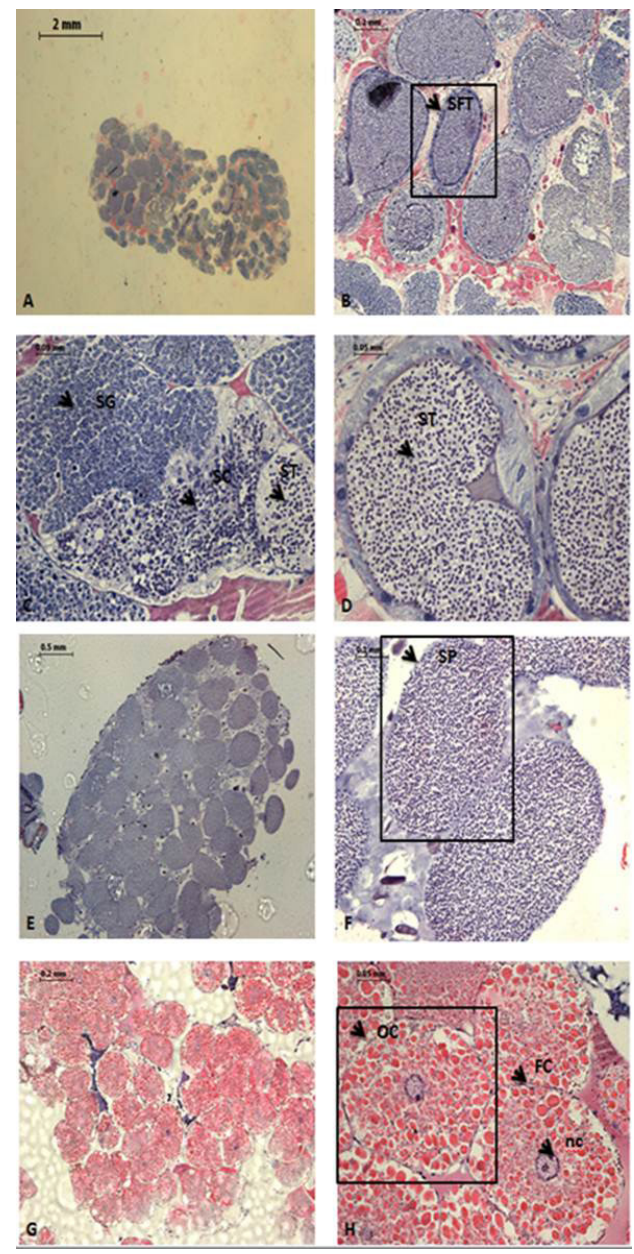

Figure 8. Hematoxylin-eosin staining of Eriocheir sinensis reproductive tissues. (A) Integrity testes. (B) Seminiferous tubule (SFT). (C) Testes containing different staged cells (SG: spermatogonium; SC: spermatocyte; ST: spermatid). (D) Seminiferous tubule filled with spermatids. (E) Integrity seminal vesicle. (F) Spermatophore (SP). (G) Integrity ovary. (H) Oocyte (OC), follicular cell (FC), and nucleus (nc).

Western blotting confirmed the presence of Es-DDX6 in solubilized protein extracts from testis and ovary tissues protein (Figure 9). An immunohistochemistry analysis was performed to determine the temporal and spatial expression patterns of Es-DDX6 protein in the male and female reproductive systems. In the testis, Es-DDX6 was highly expressed in the cytoplasm of the spermatogonia and primary spermatocyte, but its expression decreased in the spermatids (Figure 10A-E). As these cells matured along the male reproductive system to form mature spermatids, there was no apparent expression of Es-DDX6 in the vas deferens and seminal vesicle (Figure 10F-K). In the ovary, Es-DDX6 was highly expressed in the cytoplasm of oocytes (Figure 11A), while control sections showed no specific staining (Figure 11B). Together, these data provide evidence that Es-DDX6 functions in the cytoplasm of spermatogonia, primary spermatocytes, and oocytes. 


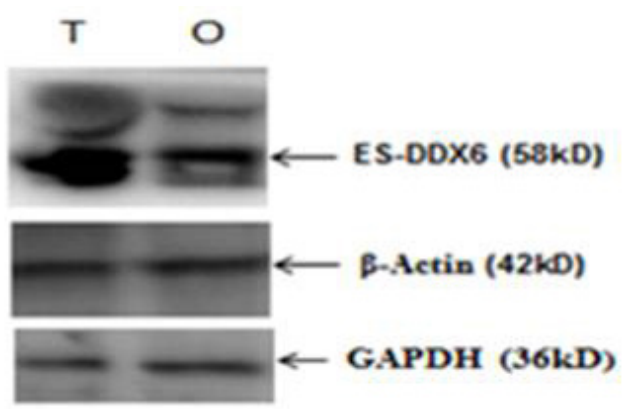

Figure 9. Western blot analysis of DDX6 protein expression levels in testes $(\mathrm{T})$ and ovary $(\mathrm{O})$ using anti-DDX6 antibodies. $\beta$-actin and GAPDH are loading controls.

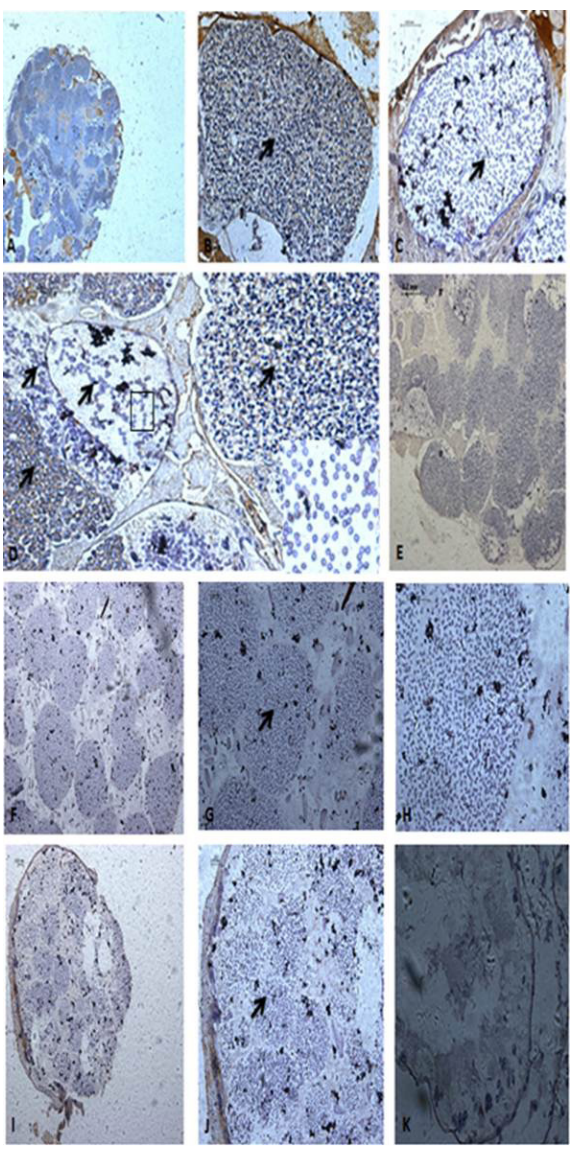

Figure 10. Immunohistochemical analysis of the distribution of Es-DDX6 protein in the male reproductive system of Eriocheir sinensis. (A) Integrity testes. (B) Seminiferous tubule. Arrowhead: primary spermatocyte. (C) Seminiferous tubule filled with spermatids. Arrowhead: spermatids. (D) Seminiferous tubule containing different staged cells. Positive immunoreactivity stains brown. Spermatogonium: strong positive immunoreactivity; spermatocyte: light positive immunoreactivity; spermatid: hardly any positive immunoreactivity. The inset in $\mathbf{D}$ is higher magnification of the boxed areas. (E) Negative control. (F and G) Seminal vesicle (no positive immunoreactivity). Arrowhead: negative signal. (H) Negative control. (I and $\mathbf{J}$ ) Vas deferens (no positive immunoreactivity). Arrowhead: negative signal. (K) Negative control. 

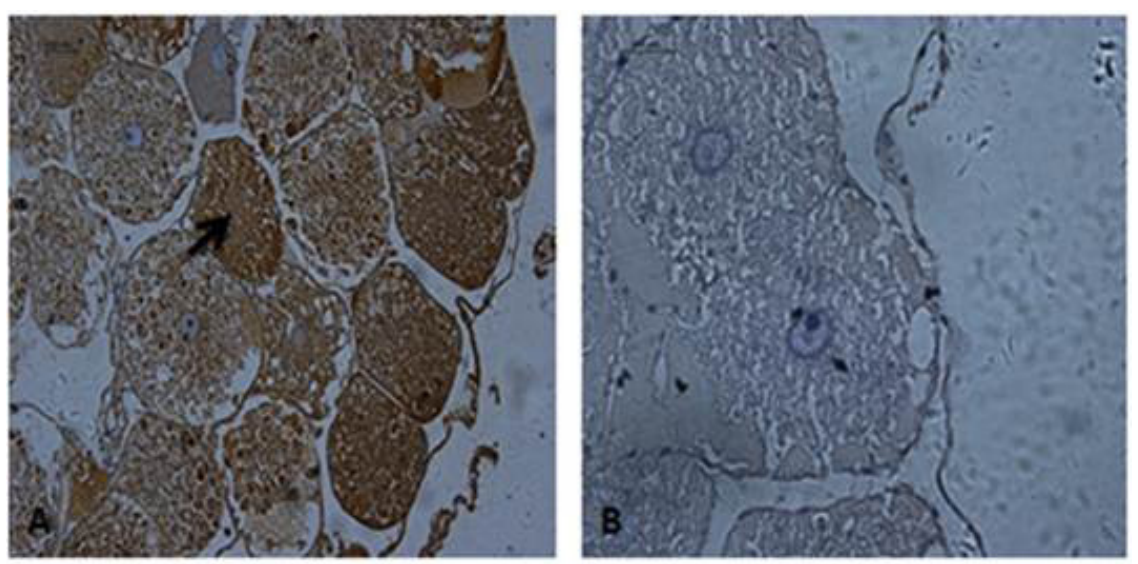

Figure 11. Immunohistochemical analysis of the distribution of Es-DDX6 protein in the female reproductive system of Eriocheir sinensis. (A) Strong positive immunoreactivity in the cytoplasm of the oocytes. (B) Negative control. Arrowhead: positive signal.

\section{DISCUSSION}

In this study, a DEAD-box family cDNA sequence (Es-DDX6) was cloned from $E$. sinensis. We have determined that Es-DDX6 contains ten conservative motifs that are classic structures observed in the DEAD-box family of proteins. Previous research has found that the Q motif was necessary for the efficient binding of ssRNA (Cordin et al., 2004). The consensus sequence for the Es-DDX6 Q motif is GWNMTTIFPQ. While the Q motif from Es-DDX6 is longer than the conservative Q motif (GACCPOHLQ), it is equal in length to Es-VASA (AKYSKPTPIQ) (Wang et al., 2012). It has been reported that motif I is critical for both ATPase and helicase activities. For example, mutations of either the first alanine residue, the conserved lysine, or the last threonine abolish ATPase activity by reducing the affinity for and the rate of hydrolysis of ATP (Rozen et al., 1989). The amino acid sequence of Es-DDX6 motif I is AKNGAGKT, with the first alanine, the lysine, and the last threonine residue being conserved, suggesting they are involved in the ATPase activity of Es-DDX6. While previous research has found that motifs Ia and Ib do not participate directly in ATP binding or hydrolysis, in association with motifs IV and V, they are essential for RNA binding. It has been speculated that alanine substitutions in motif Ia could alter both ATPase and helicase activities (Rogers et al., 2002). Motifs Ia and Ib of Es-DDX6 are PTRELA and TPGR, respectively with the alanine residue of motif Ia being conservative. Many DEAD-box proteins contain a GG doublet presents and we found it present in Es-DDX6. It has been proposed that the GG doublet motif participates in protein-protein interactions in eIF4A, and mutations of these glycines in the yeast eIF4A may affect growth (Schmid and Linder, 1991). Therefore, it was suggested that the GG doublet participated in the regulation of growth. Motif V (TRGIDIQAV), in association with motifs Ia, Ib, and IV, is proposed to be an RNA-binding motif (Cordin et al., 2006). As well, it was proposed that motif VI (HRIGRSGR) is important for both ATPase activity and RNA binding (Caruthers et al., 2000).

Additionally, it has been proposed that some DEAD-box proteins possess an annealing activity and RGG motifs or glycine-arginine-rich domains may be responsible for this 
activity. However, Es-DDX6 lacks RGG motifs or glycine-arginine-rich domains, suggesting it does contain annealing activity. While the annealing and helicase activities are distinct, the combination of both may present an advantage in the formation and regulation of the functions of an RNP (Altmann et al., 1995). As well, Es-DDX6 lacks CCHC-zinc finger domains, however, Es-VASA contains two (Wang et al., 2012). It has been proposed that CCHC-zinc finger domains are responsible for giving rise to the germ line in Strongylocentrotus purpuratus (Gustafson et al., 2011). Importantly and consistent with other members of the DEAD-box family of proteins, Es-DDX6 contains ATPase, RNA binding, and helicase activities.

To gain insight into the specific function of Es-DDX6, we determined the expression pattern of Es-DDX6 in E. sinensis. Prior research has shown that one DEAD-box helicase functioned specifically in either mouse male or female germ cells, but not both (Rocak and Linder, 2004). For example, embryonic RNA helicase is expressed only in oocytes (Sowden et al., 1995), PL10, P68, the mouse homolog of VASA Mvh, and GRTH/DDX25 are mainly expressed in male germ cells (Leroy et al., 1989; Lemaire and Heinlein, 1993; Fujiwara et al., 1994; Tang et al., 1999). Our mRNA tissue analysis demonstrates that Es-DDX6 is present in both female and male germ cells, suggesting its importance in the regulation of $E$. sinensis germ cells.

In many animals, gametogenesis involves characteristic RNA-protein particles. Pbodies containing mRNA and proteins are present in the germline cells throughout the $C$. elegans life cycle (Schisa et al., 2001). Oocyte growth is accompanied by the loss of P-bodies and a subcortical accumulation of several RNA-binding proteins, including DDX6, CPEB, and YBX2 (MSY2). These RNA-binding proteins are essential for the integrity of the P-body as depletion of any of them results in the loss of P-bodies (Eulalio et al., 2007a,b). Since previous research has shown that mutations of the GG doublet and motif $\mathrm{V}$ affected the growth of yeast (Schmid and Linder, 1991; Chang et al., 1998), we speculate that Es-DDX6 may play a role in the regulation of oocyte growth via P-bodies.

It was reported that ste13, the DDX6 ortholog of Schizosaccharomyces pombe, is required for entry into meiosis (Maekawa et al., 1994) and the levels of the C. elegans ortholog CGH-1 increase in the germ cells as they enter meiosis (Navarro et al., 2001). In the present study, we found the expression pattern of Es-DDX6 consistent with that of rck/p54, the mouse ortholog of DDX6 (Matsumoto et al., 2005). However, there was no detectable expression of Es-DDX6 in the vas deferens and seminal vesicle, which are filled with premature sperms. Since spermatocytes and oocytes are active in meiosis, it suggests that Es-DDX6 plays conserved and essential roles in E. sinensis oogenesis and spermatogenesis. Furthermore, it has been reported that the inhibition of CGH-1 expression by RNA interference results in the production of non-functioning sperm (Navarro et al., 2001). Therefore, we speculate that EsDDX6 may participate in the spermatid maturing process and sperm surveillance mechanisms. On the other hand, due to the specificity of E. sinensis spermatogenesis and since it occurs in batches, Es-DDX6 may need to continuously accumulate for the later stages of spermatogenesis, thus explaining the observed peak expression of Es-DDX6 mRNA in October. However, confirming this requires further investigation.

In conclusion, we confirmed the existence of a DDX6 ortholog in the Chinese mitten crab. The temporal and spatial distributions combined with the structural analysis reveals that Es-DDX6 plays a role in ovary and testis development, specifically in the oocyte growth process. Additionally, our immunohistochemistry results further reveal that Es-DDX6 mainly participates in the regulation of meiosis in gametogenesis. The predicted size of the Es-DDX6 
is $58.1 \mathrm{kDa}$, which is slightly bigger than the vertebrate rck/p54 $(54 \mathrm{kDa})$ in mice. In brief, this study provides a foundation for further research into the functions of E. sinensis DDX6 in gametogenesis.

\section{ACKNOWLEDGMENTS}

Research supported by grants from the National Natural Science Foundation of China (\#31201974 and\#31172393), the Shanghai Natural Science Fund Committee (\#12ZR1408900), and the Doctoral Fund of Ministry of Education (\#20120076120011). Thank for the support of the public experimental platform of the College of Life Science in East China Normal University.

\section{REFERENCES}

Abou HA and Tulsiani DR (2000). Mammalian sperm acrosome: formation, contents, and function. Arch. Biochem. Biophys. 379: 173-182.

Altmann M, Wittmer B, Methot N, Sonenberg N, et al. (1995). The Saccharomyces cerevisiae translation initiation factor Tif3 and its mammalian homologue, eIF-4B, have RNA annealing activity. EMBO J. 14: 3820-3827.

Assumpção TC, Francischetti I, Andersen JF, Schwarz A, et al. (2008). An insight into the sialome of the blood-sucking bug Triatoma infestans, a vector of Chagas' disease. Insect Biochem. Mol. Biol. 38: 213-232.

Caruthers JM, Johnson ER and McKay DB (2000). Crystal structure of yeast initiation factor 4A, a DEAD-box RNA helicase. Proc. Natl. Acad. Sci. U. S. A. 97: 3080-3085.

Chang TH, Latus LJ, Liu Z and Abbott JM (1998). Genetic interactions of conserved regions in the DEAD-box protein Prp28p. Nucleic Acids Res. 25: 5033-5040.

Cordin O, Tanner NK, Doere M, Linder P, et al. (2004). The newly discovered Q motif of DEAD-box RNA helicases regulates RNA-binding and helicase activity. EMBO J. 23: 2478-2487.

Cordin O, Banroques J, Tanner NK and Linder P (2006). The DEAD-box protein family of RNA helicases. Gene 367: 17-37.

Du NS, Xue LZ and Lai W (1988a). Histology of the male reproductive system in Eriocheir sinensis (Decapoda, Crustacea). Acta Zool. Sin. 34: 329-335.

Du NS, Xue LZ and Lai W (1988b). Studies on the Sperm of Chinese Mitten Handed Crab, Eriocheir sinensis (Crustacea, Decapoda) II. Spermatogenesis. Oceanol. Limnol. Sin. 19: 71-77.

Emery B, Cruz JDL, Rocak S, Deloche O, et al. (2004). Has1p, a member of the DEAD-box family, is required for 40S ribosomal subunit biogenesis in Saccharomyces cerevisiae. Mol. Microbiol. 52: 141-158.

Ernoult LM, Baconnais S, Harper M, Minshall N, et al. (2012). Multiple binding of repressed mRNAs by the P-body protein Rck/p54. RNA 18: 1702-1715.

Eulalio A, Behm-Ansmant I and Izaurralde E (2007a). P bodies: at the crossroads of post-transcriptional pathways. Nat. Rev. Mol. Cell Biol. 8: 9-22.

Eulalio A, Behm-Ansmant I, Schweizer D and Izaurralde E (2007b). P-body formation is a consequence, not the cause, of RNA-mediated gene silencing. Mol. Cell. Biol. 27: 3970-3981.

Fujiwara Y, Komiya T, Kawabata H, Sato M, et al. (1994). Isolation of a DEAD-family protein gene that encodes a murine homolog of Drosophila vasa and its specific expression in germ cell lineage. Proc. Natl. Acad. Sci. U. S. A. 91: 2258-2262.

Gustafson EA, Yajima M, Juliano CE and Wessel GM (2011). Post-translational regulation by gustavus contributes to selective VASA protein accumulation in multipotent cells during embryogenesis. Dev. Biol. 349: 440-450.

He L, Wang Q, Jin XK, Wang Y, et al. (2012). Transcriptome profiling of testis during sexual maturation stages in Eriocheir sinensis using Illumina sequencing. PloS One 7: e33735.

Hess RA and De Franca LR (2009). Spermatogenesis and cycle of the seminiferous epithelium. Adv. Exp. Med. Biol. 636: $1-15$.

Hu XY and Peng YF (2011). Update of studies on DEAD-box protein family and spermatogenesis. J. Reprod. Med. 20: 73-77.

Kumar S, Tamura K and Nei M (2004). MEGA3: integrated software for molecular evolutionary genetics analysis and sequence alignment. Brief. Bioinform. 5: 150-163. 
Ladomery M, Wade E and Sommerville J (1997). Xp54, the Xenopus homologue of human RNA helicase p54, is an integral component of stored mRNP particles in oocytes. Nucleic Acids Res. 25: 965-973.

Lemaire L and Heinlein UA (1993). High-level expression in male germ cells of murine P68 RNA helicase mRNA. Life Sci. 52: 917-926.

Leroy P, Alzari P, Sassoon D, Wolgemuth D, et al. (1989). The protein encoded by a murine male germ cell-specific transcript is a putative ATP-dependent RNA helicase. Cell 57: 549-559.

Maekawa H, Nakagawa T, Uno Y, Kitamura K, et al. (1994). The ste13+ gene encoding a putative RNA helicase is essential for nitrogen starvation-induced G1 arrest and initiation of sexual development in the fission yeast Schizosaccharomyces pombe. Mol. Gen. Genet. 244: 456-464.

Matsuoto K, Kwon OY, Kim H and Akao Y (2005). Expression of rck/p54, a DEAD-Box RNA helicase, in gametogenesis and early embryogenesis of mice. Dev. Dyn. 233: 1149-1156.

Minshall N, Thom G and Standart N (2001). A conserved role of a DEAD box helicase in mRNA masking. RNA 7: 17281742.

Nakamura A, Amikura R, Hanyu K and Kobayashi S (2001). Me31B silences translation of oocyte-localizing RNAs through the formation of cytoplasmic RNP complex during Drosophila oogenesis. Development 128: 3233-3242.

Navarro RE, Shim EY, Kohara Y, Singson A, et al. (2001). cgh-1, a conserved predicted RNA helicase required for gametogenesis and protection from physiological germline apoptosis in C. elegans. Development 128: 3221-3232.

Rangan P, DeGennaro M, Jaime-Bustamante K, Coux RX, et al. (2009). Temporal and spatial control of germplasm RNAs. Curr. Biol. 19: 72-77.

Rocak S and Linder P (2004). DEAD-box proteins: the driving forces behind RNA metabolism. Nat. Rev. Mol. Cell Biol. 5: $233-241$.

Rogers Jr GW, Komar AA and Merrick WC (2002). eIF4A: the godfather of the DEAD box helicases. Prog. Nucleic Acid Res. Mol. Biol. 72: 307-331.

Rozen F, Pelletier J, Trachsel H and Sonenberg N (1989). A lysine substitution in the ATP-binding site of eukaryotic initiation factor 4A abrogates nucleotide-binding activity. Mol. Cell. Biol. 9: 4061-4063.

Schisa JA, Pitt JN and Priess JR (2001). Analysis of RNA associated with P granules in germ cells of C. elegans adults. Development 128: 1287-1298.

Schmid SR and Linder P (1991). Translation initiation factor 4A from Saccharomyces cerevisiae: analysis of residues conserved in the D-E-A-D family of RNA helicases. Mol. Cell Biol. 11: 3463-3471.

Sowden J, Putt W, Morrison K, Beddington R, et al. (1995). The embryonic RNA helicase gene (ERH): a new member of the DEAD box family of RNA helicases. Biochem. J. 308: 839-846.

Tang PZ, Tsai MCH and Dufau ML (1999). A novel gonadotropin-regulated testicular RNA helicase. A new member of the dead-box family. J. Biol. Chem. 274: 37932-37940

Wang Q, Fang DA, Sun JL, Wang Y, et al. (2012). Characterization of the vasa gene in the Chinese mitten crab Eriocheir sinensis: A germ line molecular marker. J. Insect Physiol. 58: 960-965.

Xue LZ, Du NS and Lai W (1987). Histology of female reproductive system in Chinese mitten-handed crab, Eriocheir sinensis (Crustacea, Decapoda). J. East China Norm. Univ. Natur. Sci. Ed. 3: 88-97.

Zhao J, Song L, Li C, Ni D, et al. (2007). Molecular cloning, expression of a big defensin gene from bay scallop Argopecten irradians and the antimicrobial activity of its recombinant protein. Mol. Immunol. 44: 360-368. 